\title{
A Revised System of Influenza Virus Nomenclature
}

\section{A Report of the WHO Study Group on Classification ${ }^{1}$}

In 1953 the WHO Expert Committee on Influenza (2) recommended that influenza virus strains be placed into types $A, B$, and $\mathrm{C}$ on the basis of their ribonucleoprotein antigens. It was further recommended that influenza viruses be designated by a uniform code system such as A/England/1/53, where A refers to the type, England to the place of origin, 1 to the strain serial number, and 53 to the year of isolation. In 1959, a WHO Expert Committee reemphasized the importance of a uniform code system and made provisions for designating influenza subtypes based on the character of their hemagglutinin antigen, for example, A2/Singapore/ $1 / 57$ (3). The nomenclature of influenza $A$ viruses of animals followed a similar pattern with the type-specific designation and the species of origin. A subtype designation was often included, for example, A/Equine-2/ Miami/1/63.

It has now been established that the surface of the influenza virus contains an additional virus-coded antigen, the neuraminidase, which is morphologically and immunologically distinct from the hemagglutinin. The hemagglutinin and neuraminidase are known to undergo independent antigenic variation. Recognition of these changes is esscntial to our understanding of immunity. Therefore, an adequate description of the influenza viruses required that both these antigens be taken into account.

The system recommended in 1953 also implied that the antigens of the influenza viruses were unique for strains isolated from a single animal species. Hemagglutinin and neuraminidase antigens related to those of certain human influenza $A$ viruses have now been identified among strains isolated from nonhuman hosts. Viruses antigenically identical to the pandemic virus of 1968 have

\footnotetext{
1 This is an abbreviated version of the original memorandum (1).
}

also been isolated from swine and other mammals. There was no provision in the framework of the 1953 nomenclature system for the expression of such relationships which may have important implications in the epidemiology of influenza.

A study group sponsored by the World Health Organization met from 30 September to 1 October 1971 , to consider the revision of the 1953 scheme of nomenclature. The study group recommended that insofar as possible the basis of the 1953 system be retained, but that it be modified to include additional information leading to a more comprehensive description of the virus.

The recommended system consists of two parts: a strain designation, and a description of the hemagglutinin and neuraminidase antigens. The strain designation contains the following information:

1. A description of the antigenic type of ribonucleoprotein $(\mathrm{A}, \mathrm{B}$, or $\mathrm{C})$

2. The host of origin. This is not indicated for strains isolated from man but is indicated for all strains isolated from nonhuman hosts, e.g., swine, equine, duck, chicken, turkey, quail, tern, etc.

3. Geographic origin

4. Strain number

5. Ycar of isolation

The antigenic description follows the strain designation and includes, in parentheses, the following information:

1. An index describing the antigenic character of the hemagglutinin subtype, examples:

$$
\begin{array}{ll}
\text { human } & \text { H0, H1, H2, H3 } \\
\text { equine } & \text { Heq1, Heq2 } \\
\text { swine } & \text { Hsw1 } \\
\text { avian } & \text { Hav1, etc. }
\end{array}
$$

2. An index describing the antigenic character of the neuraminidase subtype, examples:

$$
\text { human } \mathrm{N} 1, \mathrm{~N}_{2}
$$




$$
\begin{array}{ll}
\text { equine } & \text { Neq1, Neq2 } \\
\text { avian } & \text { Nav1 }
\end{array}
$$

The $\mathrm{H}$ and $\mathrm{N}$ designations are intended to indicate the possession of a common subtype of hemagglutinin or neuraminidase antigen. It is implicit that a given $\mathrm{H}$ or $\mathrm{N}$ subtype will encompass strains showing a considerable degree of antigenic variation within the subtype.

The host designations of the $\mathrm{H}$ and $\mathrm{N}$ (e.g., Heq1, Nav1) antigens are based on the origin of the virus where the antigen was first characterized. It does not imply phylogenetic or evolutionary relationships between viruses containing a common $\mathrm{H}$ or $\mathrm{N}$ designation.

According to this system the full designation of a given isolate would be expressed as in the following examples:

1. $\mathrm{A} / \mathrm{PR} / 8 / 34(\mathrm{H} 0 \mathrm{~N} 1)$

2. A/Singapore $/ 1 / 57(\mathrm{H} 2 \mathrm{~N} 2)$

3. A/Hong Kong/1/68(H3N2)

4. A/turkey/Wisconsin/1/66(Hav5N2)

5. A/duck/Ukraine/1/63(Hav7Neq2)

6. A/swine/Taiwan/1/70(H3N2)

Thus, the above descriptions indicate that the influenza viruses isolated from the 1957 Asian pandemic, the 1968 Hong Kong pandemic, and from turkeys in Wisconsin all contain related neuraminidase antigens but unrelated hemagglutinins. The virus isolated from ducks in the Ukraine is seen to possess a neuraminidase antigenically similar to the neuraminidase first described for an equine influenza virus. It also indicates that the virus isolated from swine in Taiwan contains both hemagglutinin and neuraminidase antigens related to those of the human IIong Kong/68 isolate.

No provision was made for describing $\mathrm{H}$ and $\mathrm{N}$ subtypes of influenza $\mathrm{B}$ and $\mathrm{C}$ viruses. The existence of antigenic variation among strains is recognized but inadequate information exists to enable division into subtypes. The description of these viruses is, therefore, limited to strain designation, e.g., $\mathrm{B} /$ England $/ 5 / 66, \mathrm{C} /$ Paris $/ 1 / 67$.
Recombinant influenza viruses have become widely used in laboratory studies and a number of different systems have been employed to describe them. To avoid confusion a standardized system of nomenclature was recommended.

Genetically stable recombinants of influenza A viruses may be produced that contain the hemagglutinin derived from one parent and the neuraminidase from the other (antigenic hybrids). Consequently the antigenic composition of a recombinant virus containing the hemagglutinin antigen from $\mathrm{A} / \mathrm{Bel}(\mathrm{H} 0 \mathrm{~N} 1)$ and the neuraminidase from $A /$ Singapore $/ 1 / 57$ (II2N2) would be: $\mathrm{A} / \mathrm{Bel} / 42(\mathrm{H} 0)$-Singapore/1/57(N2).

For tabular material or for reference in the text of publications this description may be abbreviated to A/Bel(H0)-Sing(N2).

The division of influenza virus isolates into types $\mathrm{A}, \mathrm{B}$, and $\mathrm{C}$ is based on the complement-fixation test performed with reference sera containing antibody to the appropriate ribonucleoprotein (soluble) antigen.

The hemagglutinin antigens are divided into subtypes based on the results of hemagglutination-inhibition tests. The neuraminidase antigens are divided into subtypes on the basis of the results of neuraminidase-inhibition tests. The revised system of influenza nomenclature requires that both these antigens be characterized independently. As far as is technically possible, the use of monospecific sera prepared against isolated hemagglutinin and neuraminidase antigens derived from appropriate reference strains should be used.

The appropriate reference strain for each antigenic subtype and a more complete description of methods of antigenic analysis are published elsewhere (1).

\section{REFERENCES}

1. Bull.W.H.O. 45, 119 (1971).

2. World Health Organ. Tech. Rep. Ser. 64 (1953).

3. World Health Organ. Tech. Rep. Ser. 170 (1959).

LIST OF PARTICIPANTS

R. H. Chanock, National Institules of Heallh, Bethesda, $M D$

W. C. Cockburn, ${ }^{2}$ Virus Diseases Unit, WHO, Geneva, Switzerland

${ }^{2}$ Requests for reprints may be addressed to Dr. W. C. Cockburn, Virus Diseases Unit, WHO, 1211 Geneva 27, Switzerland. 
F. M. Davenport, The University of Michigan, Ann Arbor, MI

W. R. Dowdue, Center for Disease Control, Atlanta, GA

S. Fazekas de St. Groth, Commonwealth Scientific and Industrial Research Organization, Epping, New South Wales, Australia

H. FUkUMI, National Institute of Health, Tokyo, Japan

E. D. KIlbodrne, Mt. Sinai School of Medicine, New York, NY

G. C. SchiLd, National Institute for Medical Research, London, England

J. L. Schulman, Mt. Sinai School of Medicine, New York, NY

R. Sohier, Laboratoire National de la Sante Publique, Lyon, France

V. D. Soloviev, Gamaleya Institute, Moscow, USSR

B. Tumova, Institute of Hygiene and Epidemiology, Prague, Czechoslovakia

R. G. Webster, St. Jude Children's Research Hospital, Memphis, TE

L. JA. ZAKSTEL'SKaJA, Ivanovskij Institute of Virology, Moscow, USSR

V. M. ZDANOv, Ivanovskij Institute of Virology, Moscow, USSR

Accepted December 9, 1971 